participants with milder asthma with normal or elevated BMI. Suboptimal physical activity profile is a treatable trait which should be targeted to improve asthma-related outcomes in appropriate patients.

Please refer to page A191 for declarations of interest related to this abstract.

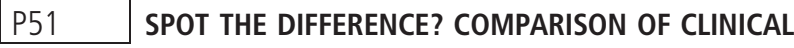 CHARACTERISTICS OF PATIENTS WITH INDUCIBLE LARYNGEAL OBSTRUCTION (ILO) AND ASTHMA REFERRED TO A SEVERE ASTHMA AND AIRWAYS TERTIARY CENTRE}

${ }^{1} \mathrm{C}$ Slinger, ${ }^{2} \mathrm{R}$ Slinger, ${ }^{1} \mathrm{~K}$ Prior, ${ }^{1} \mathrm{~A}$ Vyas, ${ }^{1} \mathrm{P}$ Mannion. 'Lancashire Teaching Hospitals NHS Trust, Preston, UK; ${ }^{2}$ Lancaster University, Lancaster, UK

\subsection{6/thorax-2021-BTSabstracts. 161}

Introduction and Objectives Patients referred our tertiary airways service are assessed for potential causation of complex breathlessness, including differential diagnosis of asthma and inducible laryngeal obstruction (ILO).

Newman et al (1995) found associations between particular patient variables when comparing patients with asthma and ILO. Further investigation of these and other variables may aid in differential diagnosis and understanding triggers and characterisation of ILO compared to asthma.

Methods Records for 70 patients with sole diagnoses of either asthma $(n=34)$ or ILO $(n=36)$ were reviewed to investigate patterns of association between a large range of variables relating to these conditions, including demographics, co-morbidities, hospital utilisation and medical treatment. Non-parametric statistics were used to compare diagnosis against categorical, interval and ratio data relating to these variables.

Results A number of significant associations were found between diagnosis and patient characteristics, summarised in table 1 .

Results showed that certain demographic variables, co-morbidities and medical treatments differentiated these two groups.
Conclusions By continuing to characterise common clinical characteristics of ILO in comparison to asthma, it is hoped that differentiation from asthma and index of suspicion for ILO will be highlighted to help further understand this clinical condition that co-exists and can mimic asthma.

It is of particular interest in the ILO only group that documented symptoms of anxiety were lower than in the group with only asthma, which may help to dispel a common held belief that people with ILO have high anxiety burden.

\section{P52 A SYSTEMATIC REVIEW OF THE EFFECTIVENESS OF EXISTING NON-PHARMACOLOGICAL INTERVENTIONS USED TO TREAT ADULTS WITH INDUCIBLE LARYNGEAL OBSTRUCTION}

${ }^{1} \mathrm{~J}$ Haines, ${ }^{2} \mathrm{JA}$ Wingfield Digby, ${ }^{2} \mathrm{~J}$ King, ${ }^{2} \mathrm{JA}$ Smith, ${ }^{2} \mathrm{SJ}$ Fowler. ${ }^{1}$ Manchester University NHS Foundation Trust, Manchester, UK; ${ }^{2}$ The University of Manchester, School of Biological Sciences, Faculty of Biology, Medicine and Health Sciences, Manchester, UK

\subsection{6/thorax-2021-BTSabstracts. 162}

Introduction Inducible laryngeal obstruction (ILO) describes transient laryngeal closure during respiration and can cause significant morbidity. Behavioural therapy is the commonly cited treatment but efficacy is largely unknown. Before a standardised approach can be developed, the potential components of intervention and their effectiveness should be understood. Aim To synthesise the current evidence base on the effectiveness of existing non-pharmacological interventions used to treat adults with ILO.

Methods Electronic databases (MEDLINE/EMBASE/CINAHL/ PsycINFO/AMED/CENTRAL) were systematically searched, informed by a PICO framework. Search terms were agreed by expert peer consensus. Two reviewers independently screened a representative sample, with lead-author completion due to excellent inter-rater reliability. Data was extracted using a predefined piloted form. Methodological quality was appraised (blindly by two reviewers) using the Joanna Briggs Institute Critical Appraisal Tools. A narrative synthesis was performed

Abstract P51 Table 1 Patient characistics according to diagnosis

\begin{tabular}{|l|l|l|}
\hline Patient Characteristic & ILO & Asthma \\
\hline Age & Higher mean age & Lower mean age \\
\hline Gender & More females & More males \\
\hline FEV1\% predicted & Higher & Lower \\
\hline FEV1/FVC ratio & Higher & Lower \\
\hline Globus & More likely & Less likely \\
\hline Stridor & More likely & Less likely \\
\hline Dysphonia & More likely & Less likely \\
\hline Dysphagia & More likely & Less likely \\
\hline Laryngeal hypersensitivity & More likely & Less likely \\
\hline Flattened inspiratory loop & More likely & Less likely \\
\hline Wheeze & Less likely & More likely \\
\hline Biologic therapy & Less likely & More likely \\
\hline Prescribed anti-depressants & Less likely & More likely \\
\hline Anxiety & Less likely & More likely \\
\hline
\end{tabular}


Abstract P52 Table 1 Effect direction plot summarising direction of outcome domains of included studies

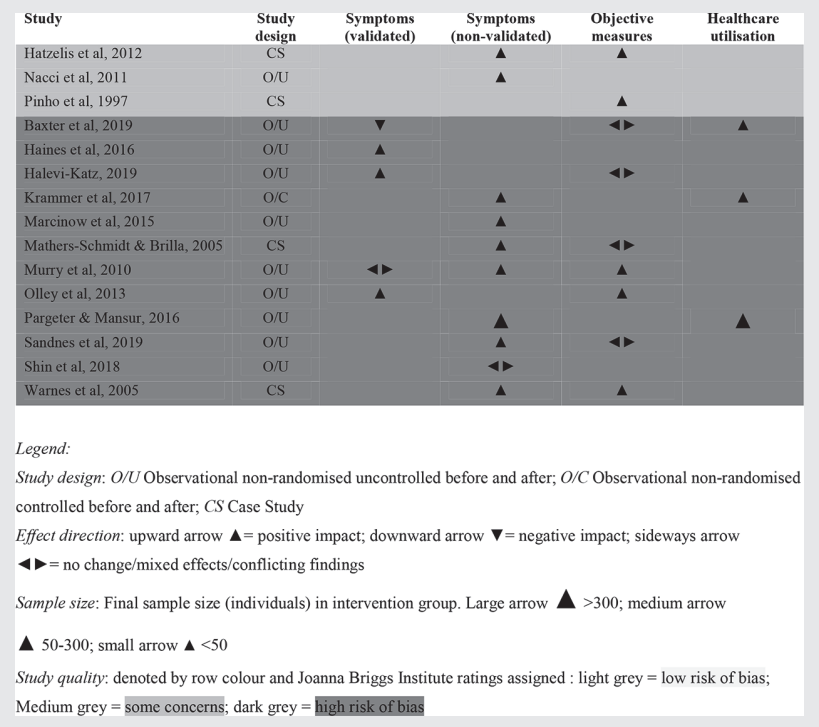

due to heterogeneity of studies (PROSPERO registration number:CRD42020213187).

Results Initial searching identified 3,359 records. Full text screening occurred in 92 records and 15 studies, comprising 555 participants, were deemed eligible. All studies were low level evidence (observational by design, with four case reports), with a high risk of bias; no studies contained control arms. Intervention description was inconsistently and poorly described but direction of effect was positive in $74 \%$ of outcomes measured (table 1). The majority of studies showed a reduction in symptom scores and improved direct laryngeal imaging post-intervention; there was an overall reduction, $59.5 \%$, in healthcare utilisation.

Conclusion The literature is in an embryonic state and lacks robust data to truly inform on the effectiveness of existing non-pharmacological interventions used to treat adults with ILO. However, positive signals in the synthesis performed support non-pharmacological treatment approaches and further development is warranted.

\section{P53 COMBINED EXPOSURE TO VAPORS, GASES, DUSTS, FUMES AND TOBACCO SMOKE INCREASES THE RISK OF ASTHMA SYMPTOMS ESPECIALLY IN ADULT- DIAGNOSED ASTHMA}

\footnotetext{
${ }^{1} \mathrm{H}$ Hisinger-Mölkänen, ${ }^{1,2}{ }^{2}$ P Pirila, ${ }^{1,2} \mathrm{~A}$ Sovijarvi, ${ }^{3} \mathrm{~L}$ Tuomisto, ${ }^{4} \mathrm{H}$ Andersen, ${ }^{5} \mathrm{~A}$ Lindqvist, ${ }^{6} \mathrm{H}$ Backman, ${ }^{7,8} \mathrm{~A}$ Langhammer, ${ }^{6} \mathrm{E}$ Rönmark, ${ }^{9} \mathrm{~B}$ Lundback, ${ }^{3,10} \mathrm{P}$ Ilmarinen, ${ }^{3,9,10} \mathrm{H}$ Kankaanranta, ${ }^{11} \mathrm{P}$ Pallasaho. ${ }^{1}$ University of Helsinki, Helsinki, Finland; ${ }^{2}$ Unit of Clinical Physiology, HUS Medical Imaging Center, Helsinki University Central Hospital, Helsinki, Finland; ${ }^{3}$ Department of Respiratory Medicine, Seinäjoki Central Hospital, Seinäjoki, Finland; ${ }^{4}$ Karolinska University Hospital, Thoracic Oncology Unit, Tema Cancer, Stockholm, Sweden; ${ }^{5}$ Clinical Research Unit of Pulmonary Diseases, Helsinki University Hospital, Helsinki, Finland; ${ }^{6}$ Department of Public Health and Clinical Medicine, Division of Occupational and Environmental Medicine/the OLIN Unit, Umeå University, Umeå, Sweden; ${ }^{7}$ HUNT Research Centre, Department of Public Health and Nursing, NTNU, Norwegian University of Science and Technology, Levanger, Norway; ${ }^{8}$ Levanger Hospital, NordTrøndelag Hospital Trust, Levanger, Norway; ${ }^{9}$ Krefting Research Centre, Institute of Medicine, University of Gothenburg, Gothenburg, Sweden; ${ }^{10}$ Faculty of Medicine and Health Technology, Tampere University, Tampere, Finland; " Espoo City Health Services, Espoo, Finland
}

10.1136/thorax-2021-BTSabstracts. 163
Background Smoking and occupational airborne exposures are known to increase asthma symptoms, but less is known about their influence by the age of asthma diagnosis.

Objective To evaluate the effect of exposures to VGDF (vapors, gases, dusts and fumes), tobacco smoke and their combination for asthma symptoms comparing subjects with asthma diagnosed in childhood and adulthood.

Methods A random sample of 16000 adults aged 20-69 years were invited to a postal survey on obstructive pulmonary diseases in Finland in 2016. Those reporting physician-diagnosed asthma and age at diagnosis were included in the analysis and their reported VGDF-exposure and smoking habits were analyzed. Age 18 years was chosen to delineate child- and adultdiagnosed asthma.

Results 8199 (51.5\%) responded. Of the responders, 831 reported physician-diagnosed asthma. 41\% of asthmatics reported child-diagnosed and 59\% adult-diagnosed asthma. Current smoking was reported by $25.2 \%$ and $20.2 \%$ and VGDF exposure by $31.3 \%$ and $44.7 \%$ in child -diagnosed and adult-diagnosed asthma, respectively. Combined VGDF-exposure and current smoking was reported by $9.7 \%$ and $10.6 \%$, respectively.

Compared to the unexposed, those with asthma diagnosed in childhood and with combined current smoking and VGDF exposure, had higher prevalence of wheeze $(69.7 \%$ vs $39.5 \%$, $\mathrm{p}=0.009)$, sputum production $(39.4 \%$ vs $11.4 \%, \mathrm{p}=0.001)$ and morning dyspnea $(42.4 \%$ vs $21.9 \%, p=0.002)$. Corresponding pattern was seen in those with asthma diagnosed in adulthood; for wheeze $(78.8 \%$ vs $53.6 \%, \mathrm{p}=0.007)$, sputum production $(40.4 \%$ vs $25.0 \%, \mathrm{p}=0.014)$ and morning dyspnea (65.4\% vs $42.0 \%, p=0.008)$. Child-diagnosed asthmatics both without exposure history $(46.5 \%$ vs $69.6 \%, p=0.001)$ and with combined exposure to smoking and VGDF $(66.7 \%$ vs 94.2\%, $\mathrm{p}=0.003$ ) reported less often $\geq 3$ symptoms compared to adult-diagnosed asthmatics, even though they reported less frequently use of asthma medication $(60.7 \%$ vs $82.0 \%$, $\mathrm{p}>0.001)$. Smoking asthmatics with adult-diagnosis and exposure to VGDF had the highest prevalence estimates of having multiple symptoms (94.2\%) in our study.

Conclusion Although asthmatics diagnosed in child- and adulthood reported symptoms related to exposure to smoking and 